\title{
Research Article \\ Sorting of Silica Nanocups by Diameter during Fabrication Process
}

\author{
Parag Deotare and Jun Kameoka \\ Department of Electrical and Computer Engineering, Texas A\&M University, 3128 College Station, TX 77843, USA
}

Received 15 August 2006; Revised 25 December 2006; Accepted 25 December 2006

Recommended by Michael Harris

\begin{abstract}
We demonstrated a new technique to sort nanoparticles based on their dimensions. Due to the interactions between charged droplets and a nonlinear electrostatic field, nanoparticles with different dimensions were deposited at different spatial locations on a given target substrate. By using this principle, we have been able to sort nanocups into three groups with mean diameters of $0.31 \mu \mathrm{m}, 0.7 \mu \mathrm{m}$, and $1.1 \mu \mathrm{m}$ and a standard deviation of $20 \%$. This technique improves the nanoparticle fabrication process not only by decreasing the standard deviation of its dimensions but also by increasing its yield since nanoparticles with different mean diameters can be generated at the same time.
\end{abstract}

Copyright (c) 2007 P. Deotare and J. Kameoka. This is an open access article distributed under the Creative Commons Attribution License, which permits unrestricted use, distribution, and reproduction in any medium, provided the original work is properly cited.

\section{INTRODUCTION}

Nanoparticles have become an important topic of research and have been developed for various applications like sensors [1], diagnostics, drug delivery [2], biological labeling [3], cosmetics [4], dyes, and so forth. The successful utilization of such applications depends on the uniformity as well as productivity of nanoparticles. Fabrication techniques such as sol-gel processing [5], chemical vapor deposition [6], self assembly [7], nanoprecipitation [8], and polymerization [9] are widely used. These fabrication processes produce particles with a broad distribution profile of their diameters. Currently, to sharpen this broad profile, several sorting techniques are utilized. However, the typical sorting process is not efficient since it is limited to low yield [10]. For instance, centrifugal separation has been used but only for large diameter particles [11]. High-pressure liquid chromatography [12], fractional crystallization [13], and gel electrophoresis [14] are some other sorting techniques. Thin film composite nanofabrication membranes [15] are also utilized to achieve sorting. These fabrication processes as well as sorting techniques produce nanoparticles either with standard deviation as large as $30 \%$ or having very low productivity. Most importantly all the processes produce only one particle distribution at a time. So, to produce different diameter particles the fabrication and sorting process has to be repeated.
To overcome these drawbacks, we present a new technique to sort nanoparticles depending on their diameter. The proper placement and orientation of the substrate used to collect nanoparticles played an important role in sorting. Since this sorting technique can be used without any extensive modification of the setup, it can be easily implemented. In addition, nanoparticles are easily removed from the substrate by ultrasonic treatment for about 15 minutes in distilled water. This process for sorting nanoparticles can yield particles for applications in drug delivery and life sciences.

\section{EXPERIMENTAL SETUP}

The experimental setup is shown in Figure 1. It consists of an electrospray source and a collection of floating electrodes as well as a counter electrode. A syringe needle is used as a source and is connected to a pump (Harvard Apparatus) to provide a constant flow of a sol-gel polymer solution. To establish a Taylor cone, a negative potential was applied between the needle and the counter electrode. The distance between the needle and the collection floating electrodes was varied to investigate the effect of deposition distance on particle diameter distribution.

A spin on glass (SOG) (Futurrex, Inc.) solution mixed with $0.3 \%$ by weight of polyvinylpyrrolidine (PVP) (Aldrich, MW 1300 000) was prepared for this experiment. Then, the 


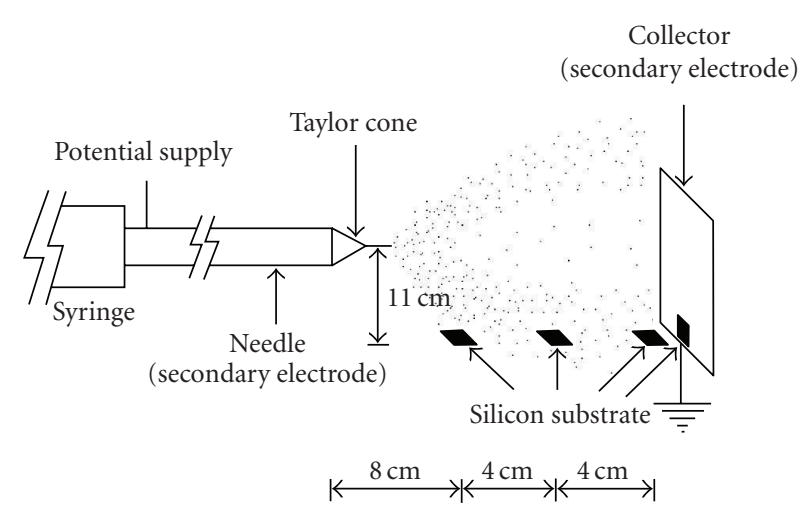

FIGURE 1: Experimental setup showing three floating electrodes, one counter electrode, and an electrospraying source that is connected to the pumping system.

solution was extruded to create nanocups [16] by the electrostatic force between the counter electrode and the source. Under this experiment, the influence of nanocup collection was studied with the counter electrode placed at a distance of $16 \mathrm{~cm}$ from the needle. A negative potential of $7.4 \mathrm{kV}$ was applied to create the electrostatic field. The silicon substrate was placed on the counter electrode, $11 \mathrm{~cm}$ below the needle axis. The reason for offsetting the substrate was that the spray does not take place along the axis of the needle but is along a conical surface with its apex at the tip of the needle. Silicon substrates were also placed as the floating electrodes, located at $8 \mathrm{~cm}, 12 \mathrm{~cm}$, and $16 \mathrm{~cm}$ from the needle. These floating electrodes were placed $11 \mathrm{~cm}$ below the needle axis but were orthogonal to the counter electrode plane as shown in Figure 1.

\section{RESULTS AND DISCUSSION}

Figure 2(a) shows a SEM micrograph of nanocups fabricated with the substrate placed on the counter electrode, $16 \mathrm{~cm}$ away from the needle and $11 \mathrm{~cm}$ below the needle axis. Cups with diameters as small as tens of nanometer to a few micrometers are collected on the substrate. This generates a very broad particle diameter distribution.

Figures 2(b), 2(c), and 2(d) show SEM images of the electrosprayed nanocups in which the distance between the counter electrode and the needle is kept constant at $16 \mathrm{~cm}$ and silicon collector substrates are placed at $8 \mathrm{~cm}, 12 \mathrm{~cm}$, and $16 \mathrm{~cm}$, respectively, as the floating electrodes. These substrates are again placed $11 \mathrm{~cm}$ below the axis of the needle and oriented orthogonal to the plane of the counter electrode. Sorting of the cups is immediately realized. Figure 2(b) shows nanocups collected on the floating electrode placed $8 \mathrm{~cm}$ away from the source. Only small cups with mean diameter around $0.31 \mu \mathrm{m}$ are collected in this case. Figure 2(c) reveals that cups with slightly larger mean diameter of $0.7 \mu \mathrm{m}$ are collected when the floating electrode is placed $12 \mathrm{~cm}$ away from the source. Finally, Figure 2(d) shows large cups with mean diameter of $1.1 \mu \mathrm{m}$ collected on the floating electrode placed $16 \mathrm{~cm}$ away from the source. The sorting of cups is obvious and we see that as the floating electrodes are moved away from the source, the percentage of larger diameter cups is increased.

The sorting mechanism in this experiment can be attributed to the total surface charges in the droplet. Because the dimension of droplet is small, about $1 \mu \mathrm{m}$ range, gravitational force [17], which is proportional to mass, is neglected. Thus, the deposition distance is determined strictly by the propelling coulombic force strength and an opposing air viscous drag acting on the droplet. The coulombic force $\left(F_{c}\right)$ on the droplets in transit is given by

$$
F_{c}=q E
$$

$E$ is the electrostatic force and $q$ is the total charges in the droplet and they are proportional to the radius cubed, that is, the volume of the particle

$$
q \propto r^{3}
$$

The viscous drag force $\left(F_{d}\right)$ is proportional to the area of the particles

$$
F_{d} \propto r^{2}
$$

Hence, under the influence of an electrostatic field the larger droplets travel longer distances and are deposited further away from the electrospray source as compared to small particles which are deposited closer to the source. Also, larger drops show higher mobility (velocity per unit electrostatic field) which further aids in traveling longer distances [17]. This agrees with the results, which show that the mean diameter of the deposited particles increases as the collecting distance is increased. This holds true only up to a certain limit. With further increase in the drop diameter, the drop mass becomes dominant and adds a significant vertical component to the drop velocity. Figure 3 is a graph showing the mean diameter of nanocups as the function of substrate position and the standard deviation. The graph reflects that the sum of the three size distributions on the left results in the fourth distribution on the right. This indicates that the modified electrospraying method sorts the cups with different mean diameter along various collector distances. The mean diameter for the first three cases is $0.31 \mu \mathrm{m}, 0.7 \mu \mathrm{m}$, and $1.1 \mu \mathrm{m}$.

\section{CONCLUSION}

We have successfully modified the electrospraying technique to sort micro/nanocups based on their dimensions during the fabrication process. Particles can be sorted with different mean diameter with relatively small standard deviation and different particle distribution can be obtained at the same time. We demonstrated fabrication and sorting of nanoparticles with mean diameter of $0.31 \mu \mathrm{m}, 0.7 \mu \mathrm{m}$, and $1.1 \mu \mathrm{m}$ and a standard deviation of about $20 \%$. The simple modification makes electrospraying a very efficient method to fabricate micro/nanoparticles for various applications. 


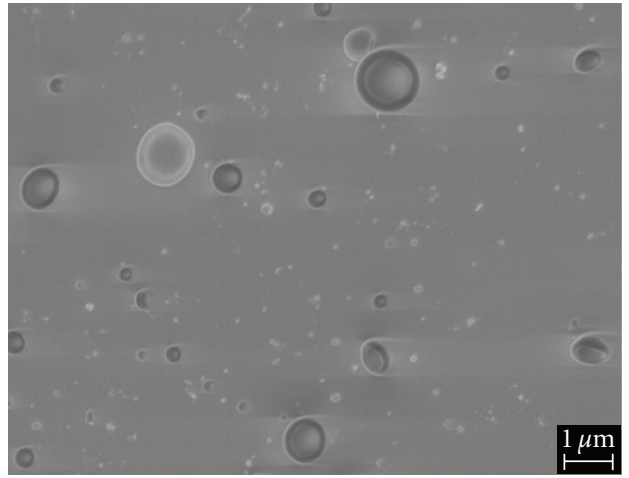

(a)

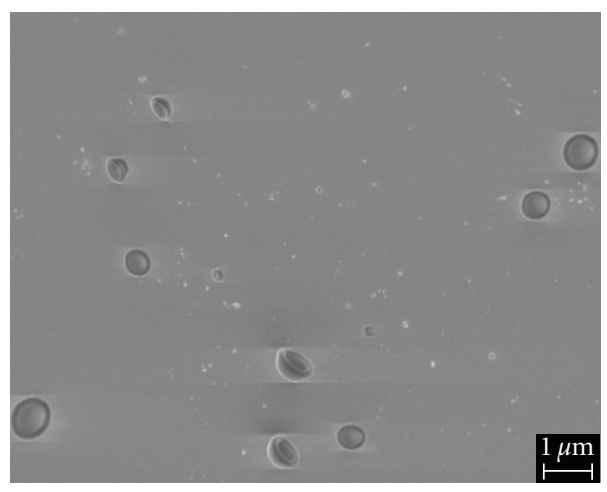

(c)

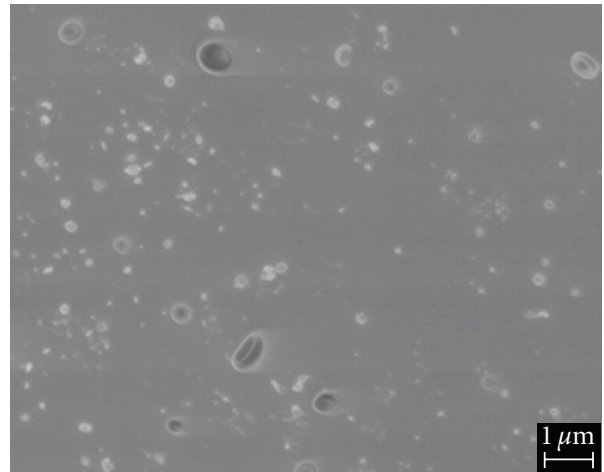

(b)

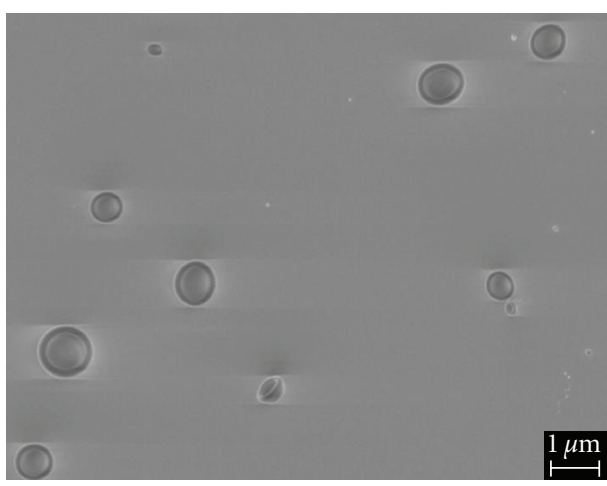

(d)

FIGURE 2: SEM images of nanocups collected at the electrodes. (a) Nanocups collected on the counter electrode that is located $16 \mathrm{~cm}$ away form the source. (b) Nanocups collected on the floating electrode $8 \mathrm{~cm}$ away from source. (c) Nanocups collected on floating electrode $12 \mathrm{~cm}$ away from the source. (d) Nanocups collected on floating electrode $16 \mathrm{~cm}$ away from the source.

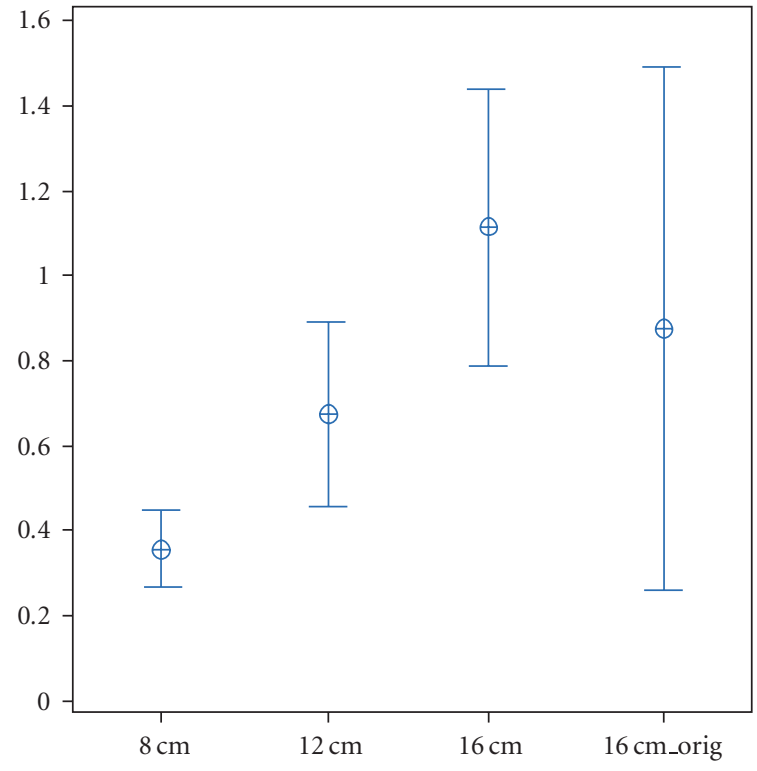

FIGURE 3: Graph showing diameter of nanocups as a function of the location of floating electrodes which are $8 \mathrm{~cm}, 12 \mathrm{~cm}$, and $16 \mathrm{~cm}$ away from the source with the counter electrode placed at $16 \mathrm{~cm}$, and $16 \mathrm{~cm}$-orig is for the nanocups collected at the counter electrode placed $16 \mathrm{~cm}$ away from the source.

\section{ACKNOWLEDGMENT}

The FE-SEM acquisition was supported by the National Science Foundation under Grant no. DBI-0116835.

\section{REFERENCES}

[1] A. N. Shipway, E. Katz, and I. Willner, "Nanoparticle arrays on surfaces for electronic, optical, and sensor applications," ChemPhysChem, vol. 1, no. 1, pp. 18-52, 2000.

[2] K. Y. Win and S.-S. Feng, "Effects of particle size and surface coating on cellular uptake of polymeric nanoparticles for oral delivery of anticancer drugs," Biomaterials, vol. 26, no. 15, pp. 2713-2722, 2005.

[3] X. He, K. Wang, W. Tan, et al., "Photostable luminescent nanoparticles as biological label for cell recognition of system lupus erythematosus patients," Journal of Nanoscience and Nanotechnology, vol. 2, no. 3-4, pp. 317-320, 2002.

[4] S. A. Wissing and R. H. Müller, "A novel sunscreen system based on tocopherol acetate incorporated into solid lipid nanoparticles," International Journal of Cosmetic Science, vol. 23, no. 4, pp. 233-243, 2001.

[5] M. Epifani, C. Giannini, L. Tapfer, and L. Vasanelli, "Sol-gel synthesis and characterization of $\mathrm{Ag}$ and $\mathrm{Au}$ nanoparticles in $\mathrm{SiO}_{2}, \mathrm{TiO}_{2}$, and $\mathrm{ZrO}_{2}$ thin films," Journal of the American Ceramic Society, vol. 83, no. 10, pp. 2385-2393, 2000. 
[6] R. A. Bennett, M. A. Newton, R. D. Smith, J. Evans, and M. Bowker, "Titania surface structures for directed growth of metal nanoparticles via metal vapour deposition and metal organic chemical vapour deposition," Materials Science and Technology, vol. 18, no. 7, pp. 710-716, 2002.

[7] R. K. Rana, V. S. Murthy, J. Yu, and M. S. Wong, "Nanoparticle self-assembly of hierarchically ordered microcapsule structures," Advanced Materials, vol. 17, no. 9, pp. 1145-1150, 2005.

[8] Y. Dong and S.-S. Feng, "Methoxy poly(ethylene glycol)poly(lactide) (MPEG-PLA) nanoparticles for controlled delivery of anticancer drugs," Biomaterials, vol. 25, no. 14, pp. 2843-2849, 2004.

[9] M. L. Hans and A. M. Lowman, "Biodegradable nanoparticles for drug delivery and targeting," Current Opinion in Solid State and Materials Science, vol. 6, no. 4, pp. 319-327, 2002.

[10] V. L. Jimenez, M. C. Leopold, C. Mazzitelli, J. W. Jorgenson, and R. W. Murray, "HPLC of monolayer-protected gold nanoclusters," Analytical Chemistry, vol. 75, no. 2, pp. 199-206, 2003.

[11] A. S. Shet, N. S. Key, and R. P. Hebbel, "Measuring circulating cell-derived microparticles," Journal of Thrombosis and Haemostasis, vol. 2, no. 10, p. 1848, 2004.

[12] J. P. Wilcoxon, J. E. Martin, and P. Provencio, "Size distributions of gold nanoclusters studied by liquid chromatography," Langmuir, vol. 16, no. 25, pp. 9912-9920, 2000.

[13] T. G. Schaaff, M. N. Shafigullin, J. T. Khoury, et al., "Isolation of smaller nanocrystal Au molecules: robust quantum effects in optical spectra," Journal of Physical Chemistry B, vol. 101, no. 40, pp. 7885-7891, 1997.

[14] T. G. Schaaff, G. Knight, M. N. Shafigullin, R. F. Borkman, and R. L. Whetten, "Isolation and selected properties of a 10.4 kDa gold: glutathione cluster compound," Journal of Physical Chemistry B, vol. 102, no. 52, pp. 10643-10646, 1998.

[15] A. Akthakul, A. I. Hochbaum, F. Stellacci, and A. M. Mayes, "Size fractionation of metal nanoparticles by membrane filtration," Advanced Materials, vol. 17, no. 5, pp. 532-535, 2005.

[16] P. Deotare and J. Kameoka, "Fabrication of silica nanocomposite-cups using electrospraying," Nanotechnology, vol. 17, no. 5, pp. 1380-1383, 2006.

[17] A. G. Bailey, Electrostatic Spraying of Liquids, edited by J. F. Hughes, SRP, Somerset, UK, 1998. 

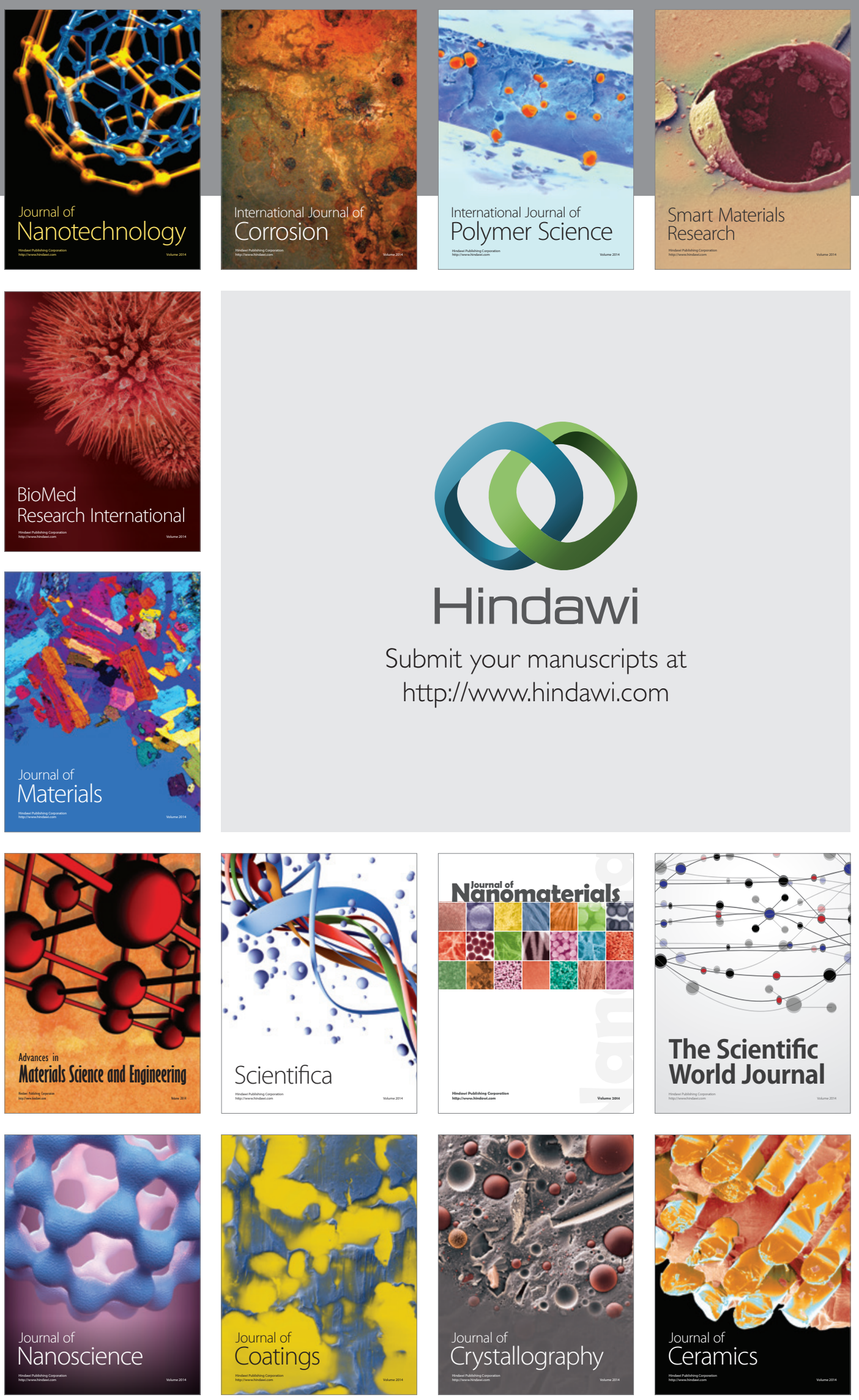

The Scientific World Journal

Submit your manuscripts at

http://www.hindawi.com

\section{World Journal}

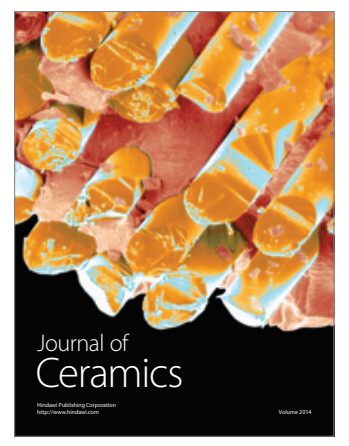

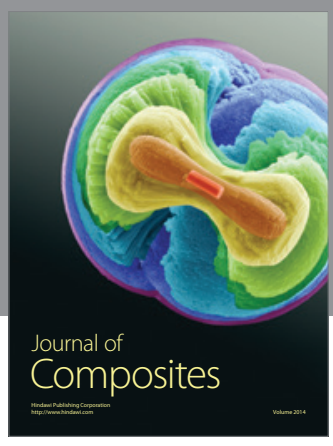
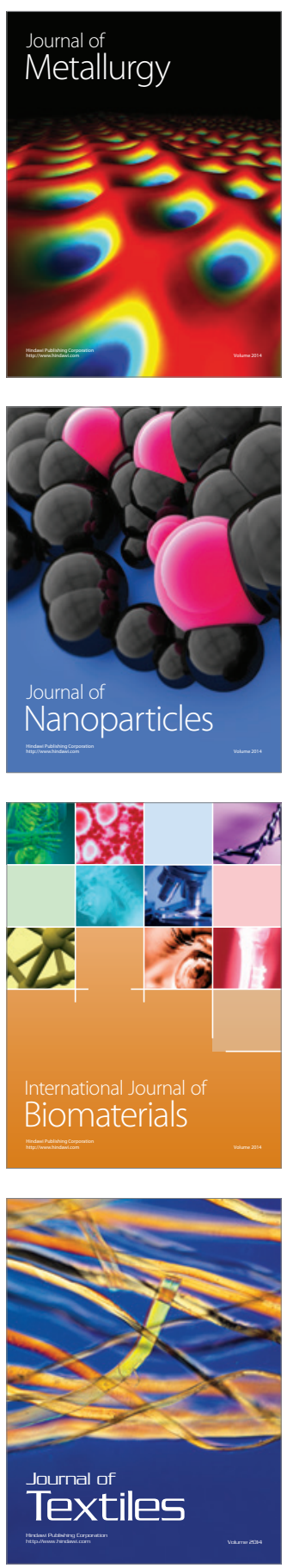